\title{
La Reforma Universitaria
}

de 1918 y la actral urascendencia

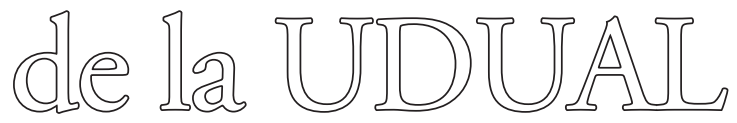

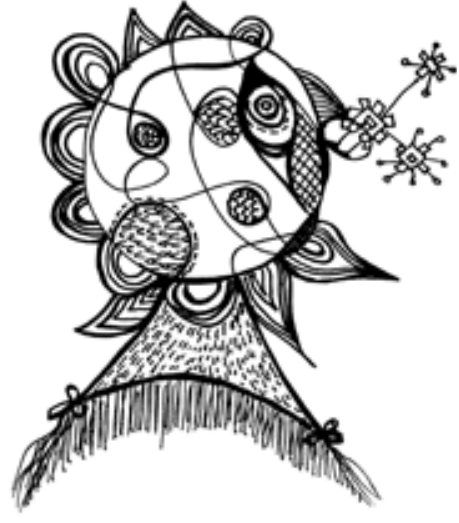

\section{Resumen}

Tras más de un siglo del llamado "Grito de Córdoba", que inició un movimiento de reforma universitaria en nuestro continente, es imperativo reflexionar sobre las nuevas luchas y debates en el campo educativo referido a los países de la región. En este artículo efectuamos una revisión de los principales ejes de la Reforma Universitaria de 1918, tomando en cuenta la necesidad de la reflexión y del estudio de nuestra propia historia universitaria ante los retos que nos propone el contexto social, científico y político actual. Al lado del análisis de un hecho histórico tan importante como la Reforma de Córdova, se efectúa a continuación la descripción general y las proyecciones del reciente encuentro de la UDUAL realizado en nuestra casa de estudios. Este organismo se constituye en una especie de radiografía permanente de lo que sucede en nuestra América Latina y el Caribe dentro del campo de la Educación Superior Universitaria.

Palabras clave: Reforma Universitaria, Grito de Córdoba, Latinoamérica, latinoamericanismo, autonomía académica, UDUAL.

\author{
Iván Rodríguez Chávez \\ Rector de la Universidad Ricardo Palma \\ rector@urp.edu.pe \\ Lima-Perú
}

Keywords: University Reform, Argentine University Reform of 1918, Latin America, Latin Americanism, academic autonomy, UDUAL.

\section{El Grito de Córdoba como inicio de una educación superior inclusiva y participativa}

\section{Introducción}

A diferencia de las universidades europeas, que fueron creación de la naciente burguesía para responder a la necesidad de incorporar nuevos y mejores conocimientos que aseguraran el poder social, político y económico, las primeras universidades en América Latina (surgidas en el siglo XVII) fueron obras del 
clero y en ellas solo se enseñaban Teología y otras ciencias ligadas a los objetivos de la Iglesia. A mediados del siglo XIX, gracias a las revoluciones liberales, hubo algunos esbozos reformistas en estos centros de Educación Superior del continente. Sin embargo, no se alcanzó una verdadera reforma. Con el paso del tiempo y aunque los fines primigenios y el espíritu de la Reforma Universitaria del 1918, en Córdova, Argentina, derivaron en nuevos anquilosamientos, esta revolución estudiantil significó una radical renovación en los fundamentos e influjos de la vida universitaria.

Resulta conveniente volver a reflexionar sobre el movimiento de Córdoba después de un siglo de su presencia, tomando en cuenta las nuevas luchas y debates en el campo de la educación en América Latina. En países como Chile, Colombia, México y Perú se han venido generando experiencias de cambios universitarios que, en algunos casos, han derivado en protestas violentas. Aires de renovación recorren, pues, el continente americano; experiencias muy distintas en toda la región parecen anunciar caminos divergentes. Por eso creemos necesaria, hoy más que nunca, la reflexión y el estudio de nuestra propia historia universitaria.

\section{Antecedentes de la reforma universitaria}

Para varios expertos, el movimiento estudiantil del 13 de diciembre de 1871 en la Universidad de Buenos Aires fue el primer antecedente importante de la Reforma Universitaria de 1918 (v. Halperin, 1972; Buchbinder, 2005; Ortiz y Scotti, 2008). Espoleada por la injusta desaprobación de un estudiante de Derecho, aquel movimiento buscaba alcanzar una verdadera reforma para asegurar la independencia y autonomía de la universidad.

Desde inicios del siglo XX se dieron una serie de hechos que también prefiguraban la revolución en el ámbito académico y social, y significaría definir el espíritu del movimiento estudiantil latinoamericano iniciado en la Universidad de Córdoba y desarrollado en el período de las guerras mundiales. Entre 1903 y 1905 se extendieron los conflictos en la Universidad de Buenos Aires, que habrían de concluir con la fundación del Centro de Estudiantes de Derecho y con la reforma del estatuto universitario. También en el Perú hubo un hecho significativo relacionado con el ámbito universitario. En 1909, en el Cuzco, "se inició una huelga estudiantil impulsada por Ángel Vega Enríquez quien proclamó, en un extenso discurso en torno a los valores del indigenismo, la funesta inmersión de la

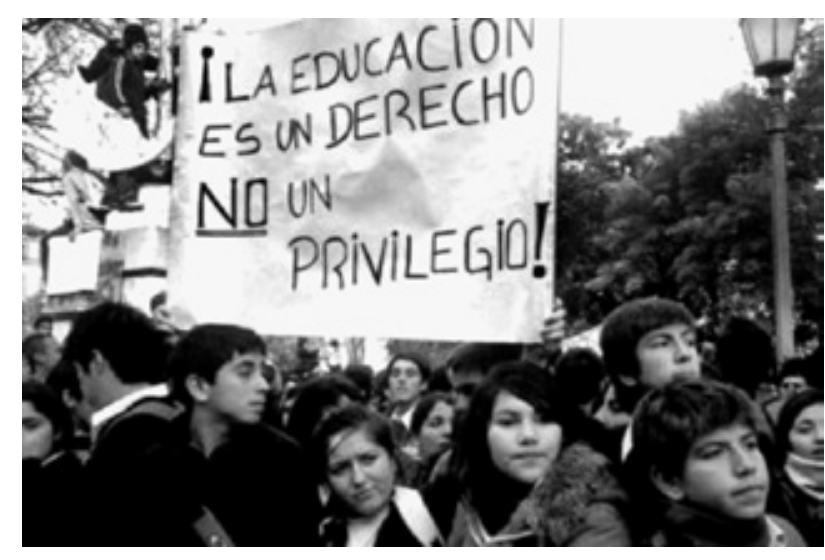

universidad en un atraso de sesgo medieval" (Negro, 2019, p. 2).

El mismo año en que se creó el primer centro de estudiantes de Derecho en Argentina, se fundaba en Chile la Federación Universitaria y dos años más tarde se desarrollaría el primer Congreso de Estudiantes en Uruguay con delegaciones de diversos países entre los que se contaba el Perú. Cuatro años después, en 1910, se llevó a cabo en Buenos Aires el Segundo Congreso de Estudiantes, al cual también asistió una delegación peruana. Los temas transversales en estos congresos estudiantiles estaban ligados a la autonomía de la universidad y a su rol en la democratización de la enseñanza. Tras el segundo congreso se conformó la Oficina Internacional Universitaria Americana con sede en Montevideo y se acordó celebrar su tercera reunión en 1912, en Lima. Esta se desarrolló

en un contexto complejo debido a la interrupción de las relaciones diplomáticas entre Perú y Chile. Estuvieron presentes los representantes estudiantiles de trece países. La buena voluntad de diálogo de los estudiantes peruanos y chilenos generó la composición de la Canción de la Juventud Americana, con la letra del peruano José Gálvez y la música del chileno Enrique Soro (Negro, 2019, p. 3).

El inicio de la Primera Guerra Mundial implicó la suspensión del siguiente congreso que había sido fijado para el año 1914 y debía llevarse a cabo en Santiago de Chile.

Asimismo, grandes hechos políticos y sociales vividos previamente en el mundo y en América Latina irradiaron también su influencia sobre los reformistas. El primero que cabe destacar es la Revolución mexicana (1910-1917) con sus demandas de justicia social, la alfabetización de la población, el reparto agrario y 
la educación para el desarrollo del país. Otro hecho importante es la Revolución rusa de 1917, que alentó en los jóvenes universitarios el anhelo social de reformas liberales y estimuló la creación de diversos partidos políticos de ideología socialista. Además, "[1]a lectura que varios intelectuales ligados a la reforma realizan de la revolución rusa hacen suponer una estrecha cercanía con el pensamiento de Lenin acerca del imperialismo" (Naidorf et al., 2018, p. 89).

\section{El Grito de Córdoba}

La tarde del sábado 15 de junio de 1918, un grupo de estudiantes de la Universidad Nacional de Córdoba irrumpió en el local del rectorado donde se celebraba la sesión de la Asamblea Universitaria e impidió el normal desarrollo de la misma. En marzo de ese año, los estudiantes habían formado el Comité Pro Reforma Universitaria y en mayo fundaron la Federación Universitaria de Córdoba.

Aquella tarde se celebraba la elección de un nuevo rector. Enrique Martínez Paz, el candidato que impulsaban los estudiantes, había sido derrotado por Antonio Nores, el candidato de la Corda Frates, una logia de aristócratas y ultraconservadores que ejercía el poder político en la provincia de Córdoba y en la universidad. Presumiblemente, la "Corda" había presionado a los votantes de un tercer candidato para que volcaran su voto en favor de Nores.

Este fue el detonante de una protesta violenta que, gracias al eficiente liderazgo de los dirigentes estudiantiles, no llegó a mayores pero se convirtió en el punto desde el cual se inició una lucha en favor de la autonomía de la universidad y en contra del encasillamiento de tipo "monárquico y monástico" de las universidades latinoamericanas a principios del siglo XX.

\section{El Manifiesto}

El viernes 21 de junio de 1918 apareció en La Gaceta Universitaria el Manifiesto Liminar, redactado por uno de los principales dirigentes estudiantiles, Deodoro Roca, y firmado por Enrique Barros, Horacio Valdés, Ismael Bordabehere, Gumersindo Sayago, Alfredo Castellanos, Luis Méndez, Jorge Bazante, Ceferino Garzón Maceda, Julio Molina, Carlos Suárez Pinto, Emilio Biagosch, Ángel Nigro, Natalio Saibene, Antonio Medina Allende y Ernesto Garzón, todos ellos dirigentes de la Federación Universitaria de Córdoba.

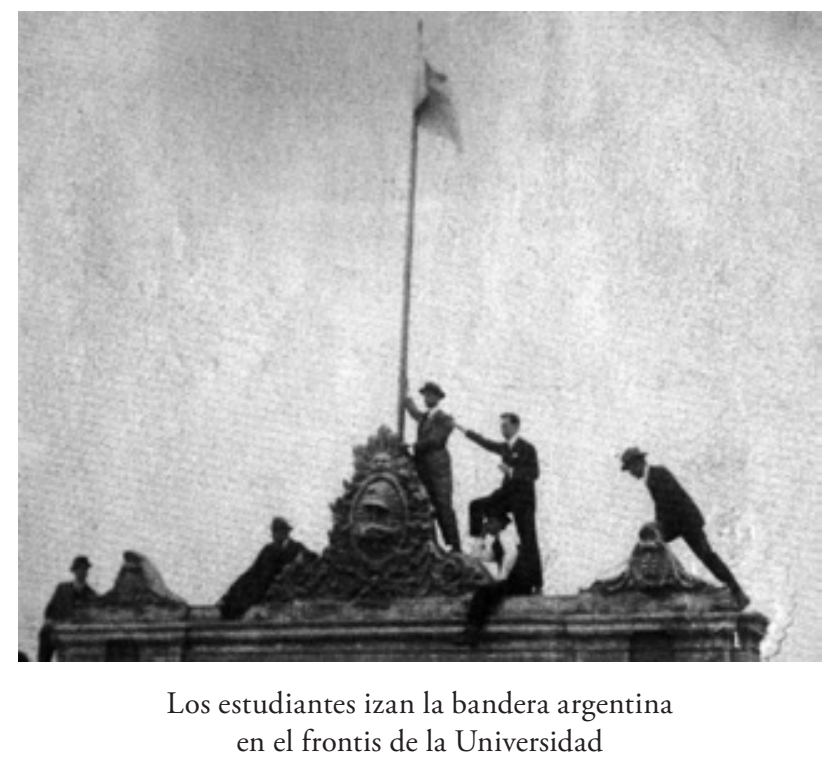

En el documento se explicaba a la opinión pública los motivos que llevaron a los estudiantes a interrumpir la elección del rector. Además, se hacía referencia a las estructuras coloniales que aún entonces ejercían poder sobre la sociedad y la universidad cordobesa y, por extensión, sobre toda latinoamericana. Su objetivo fue obtener "las libertades que faltan"; y además proyectar el movimiento a todo el continente.

Las reivindicaciones estudiantiles tuvieron tres frentes: social, organizacional y politico. En lo social promovieron la gratuidad en la enseñanza, la cátedra

\section{Aquella tarde se celebraba}

la elección de un nuevo rector.

Enrique Martínez Paz, el candidato que impulsaban los estudiantes, había sido derrotado por Antonio Nores, el candidato de la Corda Frates, una logia de aristócratas y ultraconservadores que ejercía el poder político en la provincia de Córdoba y en la universidad. Presumiblemente, la "Corda" había presionado a los votantes de un tercer candidato para que volcaran su voto en favor de Nores. 
libre y periódica, la asistencia libre, la participación en el estudio de los problemas nacionales; también se proyectó el fin de la exclusividad de las clases altas en las aulas universitarias. En lo organizacional, demandaron el establecimiento de la autonomía universitaria integral que implicaba la separación del Estado, la independencia de la Iglesia y la inviolabilidad del recinto. Finalmente, en lo político, exigieron el derecho de los estudiantes de designar a sus representantes en los órganos de gobierno. Se buscaba, con todo ello, generar el conocimiento que sirviera como instrumento de desarrollo, soberanía, independencia y libertad.

\section{Pensamiento latinoamericanista}

El proyecto continental de la Reforma Universitaria de Córdova se evidenciaría claramente desde la aparición del Manifiesto Liminar. Se destaca allí la activa participación de los jóvenes intelectuales de la época, como el cubano Julio Antonio Mella y el peruano José Carlos Mariátegui, en la tarea impulsadora y reflexiva sobre el movimiento fuera de Argentina. Precisamente, nuestro compatriota señalaría al respecto:

Todos convienen en que este movimiento, que apenas ha formulado su programa, dista mucho de proponerse objetivos exclusivamente universitarios y en que, por su estrecha y creciente relación con el avance de las clases trabajadoras y con el abatimiento de viejos principios económicos, no puede ser entendido sino como uno de los aspectos de una profunda renovación latinoamericana (2007, p. 102).

Para la investigadora mexicana Renate Marsiske Schulte, el latinoamericanismo del programa de los universitarios reformistas tuvo una coherencia no conocida antes: "Los dirigentes estudiantiles no pensaron en categorías de estados nacionales, sino consideraron a las universidades como pilares de una nueva latinoamericanidad" (en Naidorf et al., 2018, p. 93).

\section{La reforma en el Perú}

En 1919, los estudiantes de la Universidad de San Marcos Jorge Guillermo Leguía, Manuel G. Abastos, Ricardo Vegas García, José León y Bueno, Eloy Espinoza Saldaña, Jorge Cantuarias y Jorge Basadre, agrupados alrededor del magisterio de Raúl Porras Barrenechea, organizaron un ciclo de conferencias sobre el período de la Independencia (1800-1825) tomando en cuenta la proximidad del Centenario. Ese mismo año, como señala Manuel Pantigoso, "se precipitaron los acontecimientos conocidos como el Movimiento de la Reforma Universitaria. Los estudiantes buscaban reformular la enseñanza así como renovar la plana docente de la Universidad" (2019, p. 41). En su manifiesto se leía:

Por vez primera los universitarios hablan al país en nombre de la cultura. Nuestra palabra interpreta el sentimiento de la nacionalidad y el entusiasmo y la esperanza de veinte generaciones. Perseguimos la organización nacional por medio de la cultura nacional (en Pantigoso, 2019, p. 41).

Es evidente que la reforma universitaria en nuestro país se inspiró en la iniciada en Córdoba, pero sin embargo tuvo características propias. El manifiesto universitario peruano demandaba principalmente el establecimiento de cátedras libres pagadas por el Estado, la legalización del derecho de tacha a los docentes, la asistencia libre y la representación estudiantil en el Consejo Universitario. Este documento fue suscrito por José Manuel Calle, Raúl Porras Barrenechea, Víctor Raúl Haya de la Torre, Jorge Guillermo Leguía, Jacobo Hurwitz, Manuel Seoane y Luis Alberto Sánchez:

La presidencia de Augusto B. Leguía (1919-1930) significó un cierto sesgo incluyente de la clase media emergente en las instituciones políticas, si bien más adelante se transformó en un esquema de dominación oligárquica y autoritaria. La ley orgánica de enseñanza de 1920 incorporó varias de las demandas estudiantiles entre las que se hallaban que las cátedras se obtenían por concurso y duraban diez ańos, con posibilidad de volver a concursar para la misma cátedra; la creación de las categorías de catedráticos extraordinarios y docente auxiliares, así como la cátedra libre (Negro, 2019, p. 6).

En 1920, del 11 al 20 de marzo, se desarrolló en el Cuzco el Primer Congreso Nacional de Estudiantes. Víctor Raúl Haya de la Torre fue elegido como el primer presidente de la Federación de Estudiantes del Perú. Además, se aprobó la creación de las Universidades Populares. Este congreso defendió la existencia de cátedras libres, la presencia de delegados de los estudiantes en el Consejo Universitario; en lo social, se proclamó la reivindicación material y espiritual del indio; y en lo político, se dio un distanciamiento respecto al gobierno de Leguía. Además, se creó la Universidad Popular Manuel González Prada, que extendía la formación universitaria gratuita a trabajadores asalariados e individuos socialmente excluidos. La inauguración de la primera universidad popular se realizó en el Palacio de la Exposición de Lima, el 22 de enero de 1921. Luego se organizarían otras experiencias similares en 
Barranco, Callao, Arequipa, Salaverry, Trujillo, Cuzco, Ica, Chosica y Jauja.

La aparición de las universidades populares fue un hecho muy relevante puesto que entonces solo tenían derecho al voto quienes supieran leer y escribir. De ese modo, se estableció y fortaleció un inédito objetivo común entre los estudiantes y los sectores populares que derivaría en la obtención de importantes derechos laborales. Como señaló la doctora Sandra Negro en su texto de presentación de la muestra El pensamiento americanista en tiempos de la Reforma Universitaria de 1918 y su proyección en el Perú, organizada por el Rectorado de la Universidad Ricardo Palma a través de su Instituto de Investigación del Patrimonio Cultural:

Estos esfuerzos conjuntos expresaban la voluntad de cambio de una sociedad que había navegado a través de los siglos de la historia virreinal y republicana temprana, dentro del tradicionalismo y la marginación social, política y económica $(2019$, p. 6).

\section{La UDUAL, red de cooperación hacia una educación internacionalizada}

La Unión de Universidades de América Latina y el Caribe (UDUAL) es la red de universidades e instituciones de educación superior más grande y consolidada de la región. El 22 de septiembre de este año se cumplieron 70 años de su fundación, con más de 230 instituciones afiliadas de 22 países.

Fundada en 1949 en la Universidad de San Carlos de Guatemala, la UDUAL se constituyó con la finalidad de crear lazos firmes de cooperación entre las instituciones educativas de la región. Desde entonces, rectores y autoridades académicas se reúnen periódicamente para discutir, analizar y establecer importantes objetivos comunes que apunten a una educación internacionalizada, a mejorar la calidad de la enseñanza, a fomentar la investigación y la búsqueda de solución a los problemas sociales, medioambientales y culturales de la región.

Algunos de los temas fundamentales de esta organización son la internacionalización, la evaluación y la acreditación académica. Todo ello propiciado dentro de un marco de respeto y pluralidad, y con el compromiso social que la educación superior supone. Asimismo, el tema emblemático de la UDUAL ha sido la defensa de la autonomía universitaria como institución académica, con las correspondientes libertades de pensamiento y de cátedra. En ese sentido, la UDUAL defiende los valores centrales de la universidad ya presentes en la Reforma Universitaria de 1918.

La autonomía universitaria es el bien jurídico más preciado, así como lo es la responsabilidad de las universidades frente al cambio social y el cogobierno (v. Rodríguez, 2019, pp. 51-98). La reforma de 1918 dio lugar al acercamiento entre las universidades del país y entre las latinoamericanas. La UDUAL cumplió un papel clave en este campo. Desde su creación ha sido la única entidad asociativa universitaria de la región que ha vinculado a las universidades públicas más representativas, las ha orientado en su labor educativa y las ha fortalecido.

Sorprende que en pleno siglo XXI se discuta aún sobre autonomía universitaria, cuando parecía que en el siglo pasado el tema había quedado superado. Sin embargo, la defensa de la autonomía universitaria continúa y se encuentra en renovación constante (v. Centro Interuniversitario de Desarrollo, 2019). Renate Marsiske Schulte, docente de la Universidad Nacional Autónoma de México (UNAM), considera que-debido a las distintas interpretaciones que hay de este conceptoes necesario diseñar un agente de investigación sobre los textos que se refieran a la autonomía universitaria (v. 2004). Para ello es importante analizar cómo se refleja la autonomía universitaria en la vida diaria de profesores y estudiantes, qué significa la libertad de cátedra y de expresión de ideas en la universidad pública y, también, porqué la autonomía universitaria es la base para el futuro de las universidades del continente en el presente siglo.

En el seminario "2019, Año de las autonomías: reflexiones sobre la universidad y su papel en la transformación social", celebrado en agosto de este año en Ciudad de México, el secretario general de la UNAM, universidad afiliada a la UDUAL, Leonardo Lomelí, señaló: "Sin autonomía, la creación de conocimiento se ve amenazada. Es una condición necesaria para la vida de las universidades y el avance de nuestra civilización".

Por su parte, Roberto Escalante Semerena, secretario general de la UDUAL, coorganizador del encuentro, señaló la existencia de amenazas a la autonomía universitaria, agresión en algunos casos contra las universidades públicas a pesar de tratarse de una de las formas más importantes de ofrecer a la sociedad un pensamiento libre, plural y crítico, así como de enfrentar los problemas que tenemos en la región. 


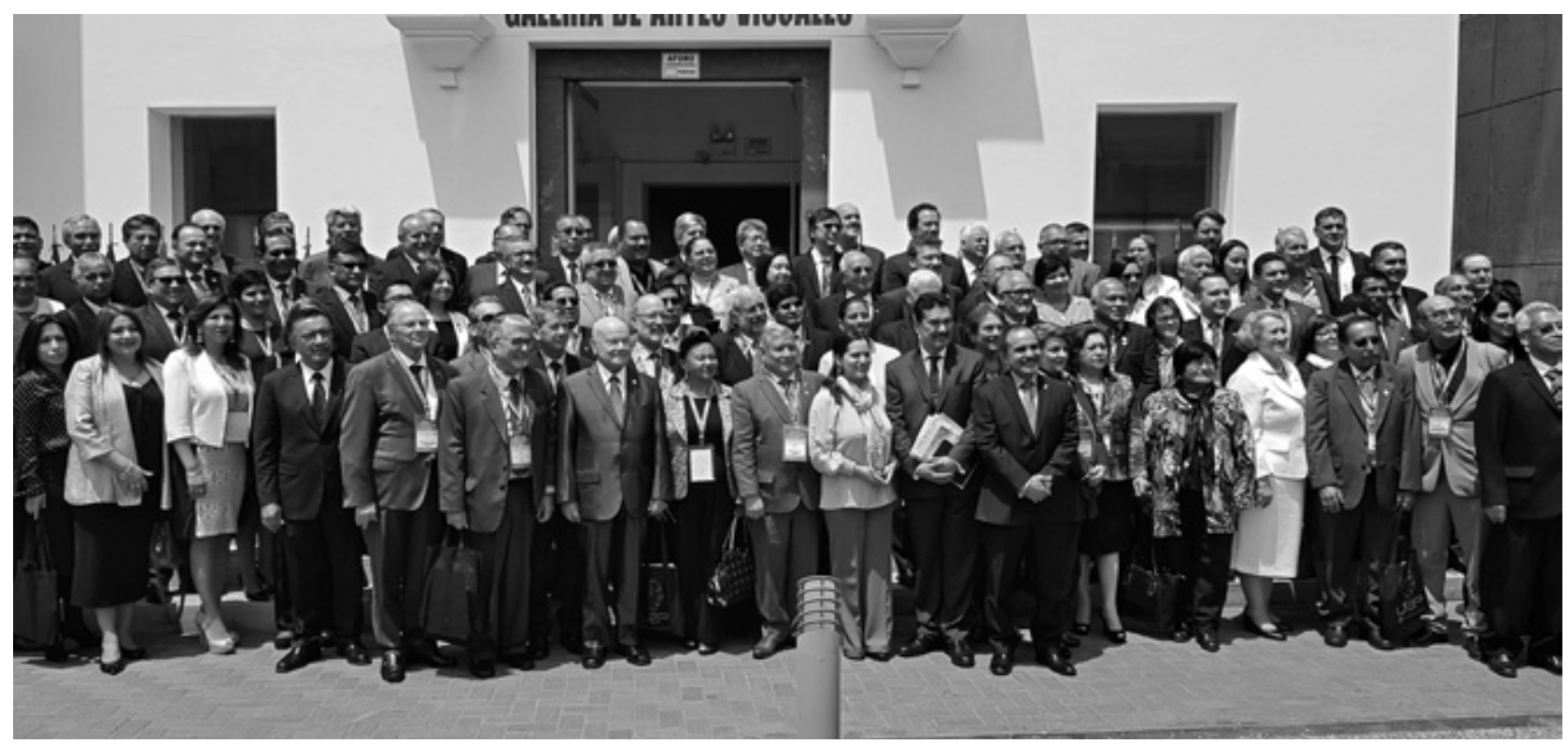

Equipo de asistentes a la UDUAL, en el frontis del Centro Cultural Ccori Wasi, de la Universidad Ricardo Palma

Otro tema importante heredero de la Reforma es, sin duda, el espíritu integrador, la búsqueda de la unión y la hermandad de todas las universidades latinoamericanas y del Caribe en pro del desarrollo igualitario en humanidades, ciencia y tecnología. Esto se manifiesta claramente en la promoción y fortalecimiento que desarrolla la UDUAL sobre la movilidad estudiantil y docente. A través de ella se ha creado una red gracias a la cual en cada semestre se permite a estudiantes cursar asignaturas en universidades de otros países inscritas en el programa. Asimismo, la movilidad de profesores para el dictado de clases, las investigaciones o publicaciones científicas producen un intercambio de conocimientos enriquecedor y estimulante. En este aspecto, un punto fundamental es la comunidad de planes de estudio a nivel latinoamericano que busca cerrar la brecha académica y facilitar los traslados e intercambios.

La Universidad Ricardo Palma, en el año de sus Bodas de Oro, ha sido sede de la XX Asamblea General de la Unión de Universidades de América Latina y el Caribe (UDUAL), celebrada el 28 y 29 de noviembre de este año ${ }^{1}$. Fue un trascendente hecho que reveló, una vez más, el compromiso firme de nuestra Casa de estudios con los valores organizacionales, científicos, tecnológicos y humanos de una alta institución como la UDUAL cuyo espíritu integrador se funda en los más elevados principios de la Reforma, reveladores de su trascendencia y vigencia en el contexto actual.

1 El periódico Propuesta, órgano periodístico de la Universidad Ricardo Palma, publicó en su edición 133 un especial sobre la XX Asamblea General de la UDUAL celebrada en nuestra casa de estudios. Puede consultarse en http://v-beta.urp.edu.pe/pdf/id/21294/n/urpespecial-2-1.pdf.
Queremos finalizar añadiendo que, definitivamente, la movilidad estudiantil y docente fue uno de los temas imprescindibles incluidos en este encuentro. Antes se decía "intercambio". A través de la UDUAL creamos una red y recibimos un mínimo de alumnos por semestre. Vienen a nuestra universidad alumnos de México, Colombia, Argentina, Ecuador y Chile. $\mathrm{Y}$ así como vienen alumnos del exterior, los nuestros también se movilizan a cualquier universidad que se haya inscrito en el programa de la UDUAL.

Otro tema importante es el intercambio de profesores para el dictado de clases y para las investigaciones o publicaciones científicas. Un tercer punto es la comunidad de planes de estudio a nivel latinoamericano a fin de cerrar la brecha y facilitar los traslados e intercambios. Al respecto, se ha logrado que la ley peruana reconozca el ejercicio de la docencia universitaria fuera del país, como si el profesor ejerciese la docencia en el Perú. Lo que se busca es cuidar el espacio común. América Latina tiene muchos aspectos que comparte, pero estamos muy fragmentados. Casi todos nos hemos formado con textos españoles, argentinos o mexicanos, pero el ideal es que todos los países puedan tener presencia en esta comunidad. Estos tres temas completarían un espectro que la UDUAL practica: la integración latinoamericana.

\section{Conclusión}

Las proclamas estudiantiles iniciadas en Córdoba en 1918 cobran una gran relevancia en el contexto actual dada la situación en la que se encuentra, de manera 


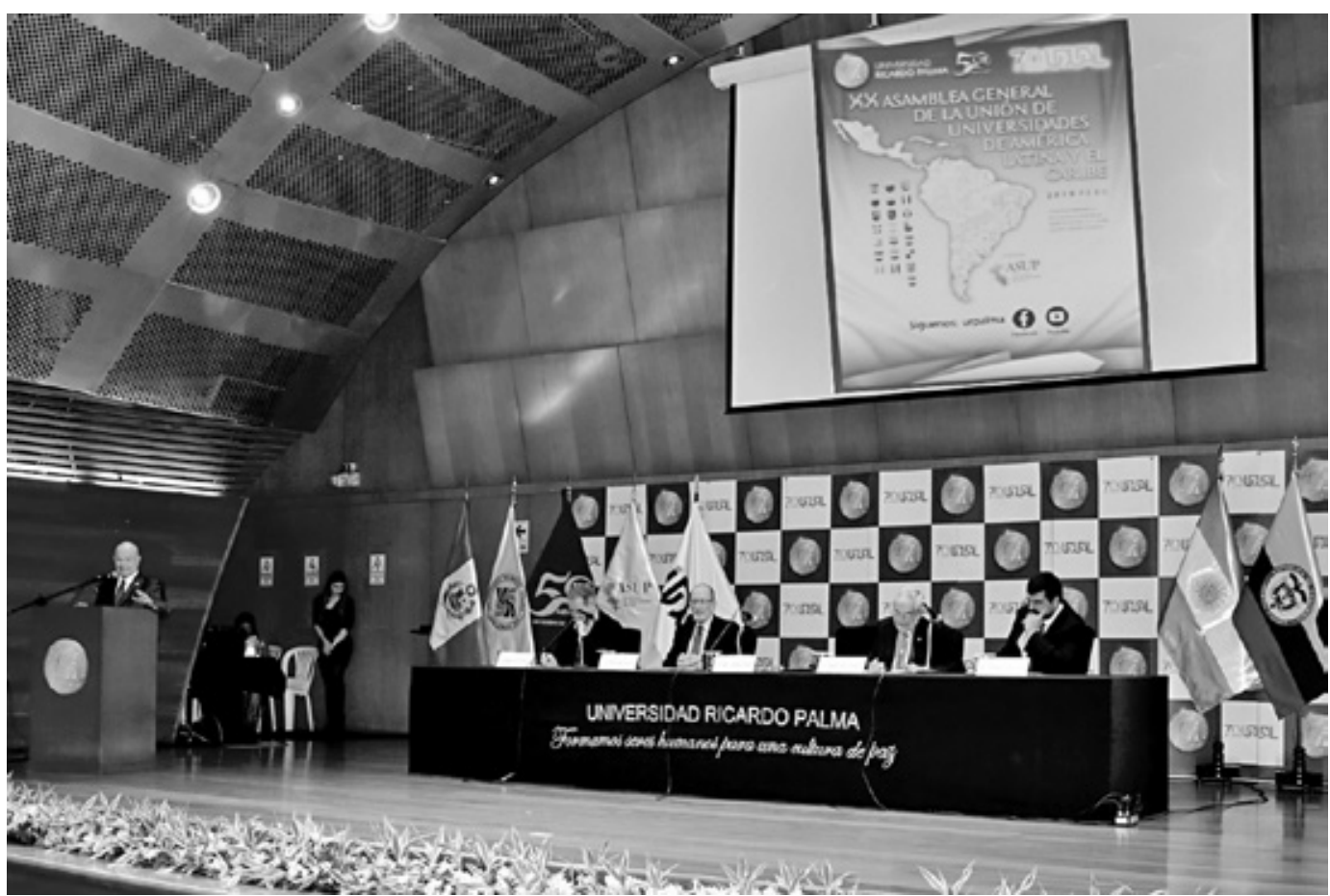

En la inauguración de la Unión de Universidades de América Latina y del Caribe (UDUAL)

general, la educación universitaria en nuestro país. La extendida presencia de universidades muestran que lo académico y lo social-cultural han cedido ante lo económico y burocrático. Por eso es conveniente y necesario recordar y enarbolar permanentemente esos pilares de la Reforma que sostienen a toda universidad: la docencia, la investigación y la extensión hacia la sociedad.

\section{Bibliografía}

Buchbinder, P. (2005). Historia de las universidades argentinas. Buenos Aires: Editorial Sudamericana.

Centro Interuniversitario de Desarrollo CINDA. (2019). Diversidad, autonomia, calidad. Desafios para una Educación Superior para el siglo XXI. María José Lemaitre, edit. Chile: RIL Editores.

Halperin Donghi, T. (1962). Historia de la Universidad de Buenos Aires. Buenos Aires: EUDEBA.

Mariátegui, J. C. (2007). 7 ensayos de interpretación de la realidad peruana. Caracas: Fundación Biblioteca Ayacucho.

Marsiske Schulte, R. (2004). "Historia de la autonomía universitaria en América Latina". En Perfiles Educativos, vol. XXVI, núm. 106, tercera época, 2004, pp. 160167, Instituto de Investigaciones sobre la Universidad y la Educación, Distrito Federal, México. Consultado en http://www.scielo.org.mx/scielo.php?script=sci_ arttext\&pid=S0185-26982004000100008 el 30 de noviembre de 2019
Naidorf, J.; Llomovate, S. y Ruiz, M. (2018). "El componente latinoamericanista de la Reforma universitaria de 1918". En Guillermo Ricca (comp.), La revolución de las conciencias. Disputas en el centenario de la Reforma Universitaria. Río Cuarto: UniRío editora, Universidad Nacional de Río Cuarto.

Negro, S. (2019). "El pensamiento americanista en tiempos de la Reforma Universitaria de 1918 y su proyección en el Perú". En la Exposición presentada en la Galería de Artes Visuales del Centro Cultural Ccori Wasi, el 28 y 29 de noviembre del 2018.

Ortiz, T. y Scotti, L. (2008). Las reformas antes de la reforma. Primeros movimientos estudiantiles en la Universidad de Buenos Aires. Facultad de Derecho. Universidad de Buenos Aires. Consultado en http://www.uba.ar/reforma/download/ reformas.pdf el 28 de junio de 2019.

Pantigoso, M. (2018). "La visión de la cultura peruana hacia el bicentenario desde la perspectiva integracionista de Abraham Valdelomar". En Tradición $\mathrm{N}^{\circ}$. 18, diciembre, pp. 139-149, Oficina del Rectorado de la Universidad Ricardo Palma, Lima, Perú.

Rodríguez Chávez, I. (2019). La universidad y la autonomía en el Perú al siglo XXI. Lima: Editorial Universitaria de la Universidad Ricardo Palma.

Schulte, R. M. (2004). "Historia de la autonomía universitaria en América Latina". En Perfiles Educativos, enero, México, 26, pp. 105-106.

Recibido el 15 de octubre de 2019 Aceptado el 25 de octubre de 2019 